\title{
Quantitative Measurements for Factors Influencing Implementation In School Settings: Protocol for A Systematic Review and A Psychometric and Pragmatic Analysis
}

\section{Sara Hoy ( $\nabla$ sara.hoy@gih.se )}

The Swedish School of Sport and Health Sciences (GIH) https://orcid.org/0000-0003-3676-8612

\section{Björg Helgadóttir}

Karolinska Institute: Karolinska Institutet

Åsa Norman

Karolinska Institute: Karolinska Institutet

\section{Protocol}

Keywords: Systematic Review, Implementation, Dissemination, Measurement, Barriers, Facilitators, Implementation Determinants, Schools, Psychometrics, Pragmatic

Posted Date: November 15th, 2021

DOI: https://doi.org/10.21203/rs.3.rs-1053472/v1

License: (c) (i) This work is licensed under a Creative Commons Attribution 4.0 International License.

Read Full License 


\section{Abstract}

Background: To address the effectiveness and sustainability of school-based interventions, there is a need to consider factors affecting implementation success. This rapidly growing field of implementation focused research is struggling with how to assess and measure implementation-relevant constructs. Earlier research identifies the need for strong psychometric and pragmatic measures. The aims of this review are therefore to (i) systematically review the literature to identify measurements of factors influencing implementation which have been developed or adapted in school settings, (ii) describe each measurement's psychometric and pragmatic properties, (iii) describe the alignment between each measurement and corresponding domain and/or construct of the Consolidated Framework for Implementation Research (CFIR).

Methods: Six databases (Medline, ERIC, Psyclnfo, Cinahl, Embase, and Web of Science) will be searched for peer-reviewed articles reporting on primary and secondary school settings, published from year 2000. Our search string will be built on three core levels of terms for: (a) implementation, (b) measurement, and (c) school settings. Two independent researchers will screen articles and extract data, with a third researcher cross-checking the process. The identified measurements will be mapped against CFIR domains and constructs, and analyzed for their psychometric and pragmatic properties using The Psychometric and Pragmatic Evidence Rating Scale (PAPERS), as well as for their measurement equivalence (invariance). The protocol follows the Preferred Reporting Items for Systematic Review and Meta-Analysis Protocols (PRISMA-P).

Discussion: By identifying measurements that are psychometrically and pragmatically strong in the field, this review can contribute to the identification of feasible, effective, and sustainable implementations strategies. Through highlighting the gaps within the range of constructs in the tools retrieved, this review may provide insights for future research and where resources could be allocated. Combined, this review will provide a greater understanding of factors influencing implementation of initiatives within school settings, and how these can be further studied.

Registration: This review is submitted to be prospectively registered on PROSPERO ID: 284741. Submission occurred the $12^{\text {th }}$ of October 2021.

\section{Background}

To date, a smorgasbord of interventions across disciplines have been designed to have an effect on different outcomes in educational settings, yet, these highly supported programmes often yield varied effects and sustainability due to poor understanding of their implementation $(1,2)$. Dissemination and implementation science (D\&l) has been on the rise especially during the last two decades. This research can be described as the study of the integration of interventions (individual or collective practices, policies, or programs) into real-world settings $(3,4)$. Studies in school-settings have consistently demonstrated that interventions are rarely implemented as designed, and that contextual factors 
affecting implementation needs to be further studied and addressed at multiple levels, from individual level to policy (5). Despite early attempts by Berman and McLaughlin (6) to question the assumption that innovations were implemented in school-settings as intended, much seems left to be addressed in the design and evaluation within school-intervention research to ensure effectiveness and sustainability of implementation $(1,5)$. The lack of studying barriers and facilitators to implementation is highlighted among reviews on school-based interventions across different outcomes, such as physical activity (7-9), tobacco or substance use (10), mental health promotion (11), and technology use among teachers in education (12), to mention a few.

Several frameworks have been developed to conceptualize and capture our understanding of how interventions are 'woven together' with a certain setting. When Durlak and DuPre (13) compiled qualitative and quantitative data on factors affecting implementation process in their review, they found strong support for 23 factors divided over five categories. The ecological framework they presented based on their results shows the vast complexity of factors influencing implementation success. These factors span over community level, to characteristics of the innovation and the provider, to organizational capacity and support systems, to specific practices, processes and staffing considerations (13). Several frameworks within implementation science address similar factors, where one of the most widely used across a wide range of studies is the Consolidated Framework for Implementation Research (CFIR) (1416). CFIR consists of 39 constructs divided over five domains; outer setting, inner setting, intervention characteristics, characteristics of individuals, and process. It is based on a spectrum of construct terminology and definitions compiled in one structured framework (17), and was designed to label and define constructs to describe contextual factors (18). The framework focuses especially on barriers and facilitators on multiple levels that may impact implementation success, similar to the ecological framework by Durlak and DuPre (13).

To study the effectiveness and sustainability of school-based interventions, there is a need to consider factors affecting implementation success. However, this rapidly growing field of dissemination and implementation focused research is struggling with how to assess and measure relevant constructs (1921). Issues concerning difficulties with understanding what variables, when, and at what level to assess, as well as the synonym, homonym, and instability of constructs being a few $(22,23)$. Studies report findings based on instruments that do not have established psychometric properties addressing their validity and reliability, which further limits their interpretability, comparability, and transferability (24). Previous research identifies the need for not only strong psychometric measures but also pragmatic measures for their likelihood of being used (25). Key criteria to pragmatic measures such as importance to stakeholders and researchers, low burden to assessors and respondents, and being actionable, should be further considered (25).

The Society for Implementation Research Collaboration (SIRC) and their Instrument Review Project (22, $26)$ is a clear example of current work within the field. To our knowledge, multiple previous reviews (20, $22,27-32$ ) have been performed to map out implementation measurements in public health and community settings, beyond health-care and clinical settings. This review contributes to expanding 
previous knowledge by assessing measurements limited to school settings as well as to capture the most recent work due to that the area of research is rapidly evolving. Therefore, the aims of this review are to (i) systematically review the literature to identify measurements of factors influencing implementation which have been developed or adapted in school settings, (ii) describe each measurement's psychometric and pragmatic properties, and (iii) describe the alignment between each measurement and corresponding domain and/or construct of the Consolidated Framework for Implementation Research (CFIR).

\section{Methods}

\section{Design and guiding frameworks}

The current review will use systematic review procedures and report in accordance with the updated Preferred Reporting Items for Systematic Reviews and Meta-Analyses (PRISMA 2020) guidelines with its extension for searches (PRISMA-S) $(33,34)$. The extension for protocols (PRISMA-P) is found for this review in Additional file 1.

Included measurements will be assessed based on recommended best practice for psychometric properties and pre-defined criteria in accordance with the Psychometric And Pragmatic Evidence Scale (PAPERS) $(35,36)$ and measurement equivalence (invariance) $(37)$. The criteria are summarized and displayed in Table 3. Further, the included measurements will be mapped against the domains and constructs of the Consolidated Framework for Implementation Research (CFIR) (17). The CFIR is one of the most widely utilized and acknowledged meta-framework within D\&I $(14,15)$, and captures factors influencing implementation success. The framework with its domains and constructs is summarized in Table 2.

This systematic review is submitted to be pre-registered on PROSPERO ID: 284741. Submission occurred the 12th of October 2021.

\section{Eligibility criteria}

Publications included will be peer-reviewed journal articles on original research, in English, and Nordic languages (Swedish, Norwegian, Danish, and Icelandic), and published from year 2000. The date restriction was chosen mainly because published work within $D \& l$ has been on the rise during the last two decades. Additionally, included publications have to report research from school settings, here including primary and secondary school, and excluding preschool, tertiary, and vocational education. This is mainly due to that the included settings are similar enough concerning organization and contextual environments. Research populations can involve school stakeholders' such as students, teachers, school leaders and management, school nurses, psychologists, assistants, and educators or similar school staff. Only quantitative measures will be included, whereas, qualitative methods will be excluded. The eligibility criteria are summarized in Table 1. 
Abstracts describing editorials, commentaries, conference abstracts, and dissertations, and other grey literature, as well as publications which reports on measures developed using exclusively qualitative methods will be judged ineligible.

\section{Search strategy}

A detailed search strategy will be developed by researchers $(\mathrm{SH}, \AA \mathrm{AN})$ together with the Karolinska Institute University Library, KIB, search specialist staff. KIB will further be enlisted to perform the searches.

To identify related measurements, we will systematically search the following six electronic databases: (i) Medline (Ovid); (ii) ERIC (ProQuest); (iii) Psyclnfo (Ovid); (iv) Cinahl (EBSCO); (v) Embase (embase.com), and (vi) Web of Science Core Collection (Clarivate). Consistent with our aim to identify and assess implementation-related measurements in the educational-, behavioral-, and health-space, the search string will be built on three core levels: (a) terms for implementation, (b) terms for measurement, and (c) terms for school settings. A test strategy for the search terms will be conducted in Web of Science Core Collection (Clarivate), then reviewed by the research team and KIB together. The final strategy will be adapted to fit the five remaining databases.

While this review mainly is situated within public health and implementation science, we will also address articles within educational science because of our chosen setting being schools. Some of the commonly used search categories from the PICO-framework (38) for systematic reviews are sometimes less useful within other types of reviews than medical, such as educational, where the outcomes included might range, control groups are not always present, and studies without an intervention are of importance to include (39). This issue will be address through searching both generic and subject specific bibliographic databases (e.g. ERIC), hand-searches, contact with experts, and citation checking (40). These additional manual searches will be performed throughout the search period, and reference lists of earlier reviews will be gone through for additional eligible articles (SH).

\section{Identification of eligible publications}

Duplicate abstracts will initially be removed by KIB (41). All remaining records will be screened in two stages. First, title and abstract will be screened independently by two researchers $(\mathrm{SH}, \mathrm{BH})$ using Rayyan software (42) according to the inclusion and exclusion criteria, where obvious irrelevant studies will be removed. Second, full-text versions of publications will be obtained for included abstracts to be screened in further detail by two researchers $(\mathrm{SH}, \mathrm{BH})$ independently. Additionally, $10 \%$ of abstracts and full texts during the two stages of screening will be cross-checked by a third researcher ( $\mathrm{A} N$ ). If a decision cannot be made regarding a full-text articles' eligibility, all three researchers will discuss the issue until consensus has been reached. A detailed description of the screening process will be presented in text and with a PRISMA flow chart of study selection figure.

Once measurements are mapped to CFIR's domains, PAPERS criteria, and measurement equivalence (invariance), we will perform the analysis of each measurement. The final set of publications included 
will be carefully evaluated for the presence of CFIR-related constructs and items, and their psychometric and pragmatic properties.

\section{Extraction of data from eligible publications}

Data will be extracted and compiled through a systematic process in accordance with a developed project codebook which covers study characteristics, PAPERS criteria, measurement equivalence (invariance), and CFIR domains by three researchers $(\mathrm{SH}, \mathrm{BH}, \AA \mathrm{AN})$. For the purpose of a similar pre-understanding of the topic, the team will read articles on related measure evaluation system (e.g., COSMIN (43)) and psychometric properties $(24,25,35-37)$, and implementation science reviews $(20,22,27)$, as well as the original work of the theoretical framework CFIR (17). As a first stage, the team will extract data according to the project codebook from two included articles, independently of each other. The similarities and differences in coding will be addressed and discussed until consensus has been reached, to initially obtain an as comparable data extraction and coding process as possible.

\section{Study characteristics}

Characteristics of each study will be extracted, synthesized and reported, such as country, setting, sample and study population, characteristics of the innovation being assessed, and guiding theoretical frameworks.

\section{CFIR coding}

Factors influencing implementation being assessed in each measurement will be coded according to CFIR's five domains and 39 constructs using a developed project codebook, based on the original work of Damschroder and colleagues (17) and data analysis tools available online (44). The coding process will focus on assessing items, constructs, and domains of each instrument and evaluate how they align with CFIR domains and constructs. Due to the considerable heterogeneity of how procedures of items, constructs, and domains are operationalized across disciplines and across researchers regarding measurement development and adaptation (29), we have chosen this three-focused coding approach. The CFIR's five domains and 39 constructs are presented in Table 2.

\section{Psychometric and pragmatic coding}

We will apply the PAPERS scale (36), and measurement equivalence (invariance) defined by Putnick and Bornstein (37), summarized in Table 3. The rating scale includes five pragmatic measurement characteristics that reflects the ease or difficulty of use. Nine psychometric measurement characteristics are included in the rating scale to assess reliability and validity All properties of the PAPERS scale are rated on six levels with predefined values; poor (-1), none (0), minimal/emerging (1), adequate (2), good (3), or excellent (5). Additionally, we have chosen to include a tenth psychometric property - invariance reflecting measurement equivalence. This is due to its importance as a prerequisite to comparing group means, and is most commonly tested through structural equation modelling using confirmatory factor analysis (37). Invariance will be descriptively assessed, and not rated against any scale. 


\section{Analysis and synthesis}

Reporting of the results will be done through both narrative descriptions as well as descriptive statistics using proportions and frequencies of the psychometric and pragmatic properties, and CFIR domains and constructs.

\section{Discussion}

This systematic review protocol gives a detailed description for identifying measurements assessing factors that influence implementation in primary and secondary school setting. It contributes in covering a fast-growing field within implementation science, where this systematic review expands on knowledge from previous reviews. Psychometric evidence is key when we want to have confidence in the results from measurements for factors influencing implementation (36). By identifying measurements that are psychometrically and pragmatically strong in the field, this review can contribute to the identification of feasible, effective, and sustainable implementations strategies. Through highlighting the gaps within the range of constructs in the tools retrieved, this review may provide insights for future research and where resources could be allocated. Another strength of this systematic review protocol is that we include psychometric equivalence (invariance) as a more contemporary framework with structural equation modelling (SEM) (37), rather than limiting us to more classical test-theory informed measurement development, a mentioned limitation of the PAPERS scale (22). Because measurement invariance is a prerequisite to comparing group means, our systematic review will give further understanding to which extent the included measurements have the ability to detect that a construct has the same meaning across groups or across measurements over time.

A limitation to our review is that we exclude abstracts describing e.g., dissertations and grey literature to detect measurements. Another limitation is that we limit our scope to factors influencing implementation rather than also including 'implementation outcomes', as other earlier reviews have done in line with the procedures of the Instrument Review Project at SIRC (22). Also, we will not include empirical research and make 'measurement packages' through citation searches done by Lewis and colleagues (22). Therefore, relevant empirical articles describing the further psychometric and pragmatic development of an original measurement may be missed. We address this limitation by including adapted measurements in our eligibility criteria, not only original developed measurement. Lastly, only articles published in English and the research teams native (Nordic) languages will be included and therefore relevant articles in other languages may be missed. The decisions for mentioned limitations are made due to pragmatic reasons, such as limited time and staff.

In sum, this review can provide a greater understanding of factors influencing implementation of innovations within educational settings and how these can be further studied. It will offer insights to which quantitative measures are easy-to-use, reliable, and valid in an emerging field of implementation science. 


\section{Abbreviations}

CFIR

Consolidated Framework for Implementation Research

CINAHL

Cumulative Index of Nursing and Allied Health Literature

COSMIN

Consensus-based Standards for the Selection of Health Measurement Instruments

D\&l

Dissemination and Implementation Science

ERIC

Education Resources Information Center

$\mathrm{GIH}$

The Swedish School of Sport and Health Sciences

KIB

Karolinska Institute University Library

PAPERS

Psychometric and Pragmatic Evidence Rating Scale

PRISMA

Preferred Reporting Items for Systematic Reviews and Meta-Analyses

PROSPERO

The International Prospective Register of Systematic Reviews

SIRC

The Society for Implementation Research Collaboration

\section{Declarations}

\section{Ethics approval and consent to participate}

Not applicable.

\section{Consent for publication}

All the authors read and approved the final protocol manuscript for publication.

\section{Availability of data and materials}

Not applicable. 


\section{Competing interests}

The authors declare they have no conflicting interests.

\section{Funding}

The current article is funded through the Knowledge Foundation, ID: 20180040, and the research project "Physical activity for healthy brain functions among school children" at the Swedish School of Sport and Health Sciences $(\mathrm{GIH})$, with professor Håkan Larsson as PI.

\section{Authors' contributions}

Review protocol methodology: SH, BH, ÅN. Eligibility criteria: SH, BH, ÅN. Search strings and terms: SH, ÅN, and KIB. Framework selection: SH, ÅN. SH wrote the first protocol manuscript draft. All authors have contributed to the final protocol manuscript.

\section{Acknowledgements}

We would like to thank Emma-Lotta Säätelä and Sabina Gillsund at KIB for performing the literature searches. Also, we would like to thank Kayne Mettert at SIRC and the Instrument Review Project for giving feedback on our search strategy, and Malin Ekstrand and Monika Janvari at GIH Library for initial feedback on our search strategy.

\section{References}

1. Moir T. Why Is Implementation Science Important for Intervention Design and Evaluation Within Educational Settings? Frontiers in Education. 2018;3(61).

2. Herlitz L, Maclntyre H, Osborn T, Bonell C. The sustainability of public health interventions in schools: a systematic review. Implementation Science. 2020;15(1):4.

3. Peters DH, Adam T, Alonge O, Agyepong IA, Tran N. Implementation research: what it is and how to do it. BMJ: British Medical Journal. 2013;347:f6753.

4. Eccles MP, Mittman BS. Welcome to Implementation Science. Implementation Science. 2006;1(1):1.

5. Lendrum A, Humphrey N. The importance of studying the implementation of interventions in school settings. Oxford Review of Education. 2012;38(5):635-52.

6. Berman P, McLaughlin MW. Implementation of Educational Innovation. The Educational Forum. 1976;40(3):345-70.

7. Cassar S, Salmon J, Timperio A, Naylor P-J, van Nassau F, Contardo Ayala AM, et al. Adoption, implementation and sustainability of school-based physical activity and sedentary behaviour 
interventions in real-world settings: a systematic review. International Journal of Behavioral Nutrition and Physical Activity. 2019;16(1):120.

8. Naylor P-J, Nettlefold L, Race D, Hoy C, Ashe MC, Wharf Higgins J, et al. Implementation of school based physical activity interventions: A systematic review. Preventive Medicine. 2015;72:95-115.

9. McHugh C, Hurst A, Bethel A, Lloyd J, Logan S, Wyatt K. The impact of the World Health Organization Health Promoting Schools framework approach on diet and physical activity behaviours of adolescents in secondary schools: a systematic review. Public Health. 2020;182:116-24.

10. Waller G, Finch T, Giles EL, Newbury-Birch D. Exploring the factors affecting the implementation of tobacco and substance use interventions within a secondary school setting: a systematic review. Implementation Science. 2017;12(1):130.

11. O’Reilly M, Svirydzenka N, Adams S, Dogra N. Review of mental health promotion interventions in schools. Social Psychiatry and Psychiatric Epidemiology. 2018;53(7):647-62.

12. Tondeur J, van Braak J, Ertmer PA, Ottenbreit-Leftwich A. Understanding the relationship between teachers' pedagogical beliefs and technology use in education: a systematic review of qualitative evidence. Educational Technology Research and Development. 2017;65(3):555-75.

13. Durlak JA, DuPre EP. Implementation matters: a review of research on the influence of implementation on program outcomes and the factors affecting implementation. Am J Community Psychol. 2008;41(3-4):327-50.

14. Birken SA, Powell BJ, Shea CM, Haines ER, Alexis Kirk M, Leeman J, et al. Criteria for selecting implementation science theories and frameworks: results from an international survey. Implementation Science. 2017;12(1):124.

15. Nilsen P. Making sense of implementation theories, models and frameworks. Implementation Science. 2015;10(1):53.

16. Kirk MA, Kelley C, Yankey N, Birken SA, Abadie B, Damschroder L. A systematic review of the use of the Consolidated Framework for Implementation Research. Implementation Science. 2016;11(1):72.

17. Damschroder LJ, Aron DC, Keith RE, Kirsh SR, Alexander A, Lowery JC. Fostering implementation of health services research findings into practice: a consolidated framework for advancing implementation science. Implementation Science. 2009;4(1):50.

18. Nilsen P, Birken SA. Handbook on implementation science. Cheltenham, UK: Edward Elgar Publishing; 2020.

19. Shah S, Allison KR, Schoueri-Mychasiw N, Pach B, Manson H, Vu-Nguyen K. A Review of Implementation Outcome Measures of School-Based Physical Activity Interventions. The Journal of school health. 2017;87(6):474-86.

20. Clinton-McHarg T, Yoong SL, Tzelepis F, Regan T, Fielding A, Skelton E, et al. Psychometric properties of implementation measures for public health and community settings and mapping of constructs against the Consolidated Framework for Implementation Research: a systematic review. Implementation Science. 2016;11(1):148. 
21. Schaap R, Bessems K, Otten R, Kremers S, van Nassau F. Measuring implementation fidelity of school-based obesity prevention programmes: a systematic review. International Journal of Behavioral Nutrition and Physical Activity. 2018;15(1):75.

22. Lewis CC, Mettert KD, Dorsey CN, Martinez RG, Weiner BJ, Nolen E, et al. An updated protocol for a systematic review of implementation-related measures. Systematic Reviews. 2018;7(1):66.

23. Gerring J. Social science methodology: A criterial framework. New York, NY, US: Cambridge University Press; 2001. xx, 300-xx, p.

24. Martinez RG, Lewis CC, Weiner BJ. Instrumentation issues in implementation science. Implementation Science. 2014;9(1):118.

25. Glasgow RE, Riley WT. Pragmatic measures: what they are and why we need them. Am J Prev Med. 2013;45(2):237-43.

26. Lewis CC, Stanick CF, Martinez RG, Weiner BJ, Kim M, Barwick M, et al. The Society for Implementation Research Collaboration Instrument Review Project: A methodology to promote rigorous evaluation. Implementation Science. 2015;10(1):2.

27. Allen P, Pilar M, Walsh-Bailey C, Hooley C, Mazzucca S, Lewis CC, et al. Quantitative measures of health policy implementation determinants and outcomes: a systematic review. Implementation Science. 2020;15(1):47.

28. Allen JD, Towne SD, Maxwell AE, DiMartino L, Leyva B, Bowen DJ, et al. Measures of organizational characteristics associated with adoption and/or implementation of innovations: A systematic review. BMC Health Services Research. 2017;17(1):591.

29. Chaudoir SR, Dugan AG, Barr CHI. Measuring factors affecting implementation of health innovations: a systematic review of structural, organizational, provider, patient, and innovation level measures. Implementation Science. 2013;8(1):22.

30. Chor KHB, Wisdom JP, Olin S-CS, Hoagwood KE, Horwitz SM. Measures for Predictors of Innovation Adoption. Adm Policy Ment Health. 2015;42(5):545-73.

31. Emmons KM, Weiner B, Fernandez ME, Tu S-P. Systems antecedents for dissemination and implementation: a review and analysis of measures. Health Educ Behav. 2012;39(1):87-105.

32. Weiner BJ, Amick H, Lee S-YD. Review: Conceptualization and Measurement of Organizational Readiness for Change: A Review of the Literature in Health Services Research and Other Fields. Medical Care Research and Review. 2008;65(4):379-436.

33. Page MJ, McKenzie JE, Bossuyt PM, Boutron I, Hoffmann TC, Mulrow CD, et al. The PRISMA 2020 statement: an updated guideline for reporting systematic reviews. BMJ. 2021;372:n71.

34. Rethlefsen ML, Kirtley S, Waffenschmidt S, Ayala AP, Moher D, Page MJ, et al. PRISMA-S: an extension to the PRISMA Statement for Reporting Literature Searches in Systematic Reviews. Systematic Reviews. 2021;10(1):39.

35. Stanick CF, Halko HM, Nolen EA, Powell BJ, Dorsey CN, Mettert KD, et al. Pragmatic measures for implementation research: development of the Psychometric and Pragmatic Evidence Rating Scale (PAPERS). Translational Behavioral Medicine. 2019;11(1):11-20. 
36. Lewis CC, Mettert KD, Stanick CF, Halko HM, Nolen EA, Powell BJ, et al. The psychometric and pragmatic evidence rating scale (PAPERS) for measure development and evaluation. Implementation Research and Practice. 2021;2:26334895211037391.

37. Putnick DL, Bornstein MH. Measurement invariance conventions and reporting: The state of the art and future directions for psychological research. Developmental Review. 2016;41:71-90.

38. Sharma R, Gordon M, Dharamsi S, Gibbs T. Systematic reviews in medical education: A practical approach: AMEE Guide 94. Medical Teacher. 2015;37(2):108-24.

39. Tai J, Ajjawi R, Bearman M, Wiseman P. Conceptualizations and Measures of Student Engagement: A Worked Example of Systematic Review. In: Zawacki-Richter O, Kerres M, Bedenlier S, Bond M, Buntins K, editors. Systematic Reviews in Educational Research: Methodology, Perspectives and Application. Wiesbaden: Springer Fachmedien Wiesbaden; 2020. p. 91-110.

40. Newman M, Gough D. Systematic Reviews in Educational Research: Methodology, Perspectives and Application. In: Zawacki-Richter O, Kerres M, Bedenlier S, Bond M, Buntins K, editors. Systematic Reviews in Educational Research: Methodology, Perspectives and Application. Wiesbaden: Springer Fachmedien Wiesbaden; 2020. p. 3-22.

41. Bramer WM, Giustini D, de Jonge GB, Holland L, Bekhuis T. De-duplication of database search results for systematic reviews in EndNote. 2016. 2016;104(3):4.

42. Ouzzani M, Hammady H, Fedorowicz Z, Elmagarmid A. Rayyan-a web and mobile app for systematic reviews. Systematic Reviews. 2016;5(1):210.

43. Terwee CB, Mokkink LB, Knol DL, Ostelo RW, Bouter LM, de Vet HC. Rating the methodological quality in systematic reviews of studies on measurement properties: a scoring system for the COSMIN checklist. Qual Life Res. 2012;21(4):651-7.

44. CFIR Research Team-Center for Clinical Management Research. Tools and Templates: Data Analysis Tools 2021 [Available from: https://cfirguide.org/tools/tools-and-templates/.

\section{Tables}

Table 1. Eligibility criteria 
i Publications from peer-reviewed journal articles on original research, in English and Nordic languages (Swedish, Norwegian, Danish, and Icelandic), and published from year 2000

ii Reported research from school settings (including primary and secondary school, excluding preschool, tertiary, and vocational education), involving school stakeholders' such as students, teachers, school leaders and management, school nurses, psychologists, assistants, and educators or similar school staff

iii Reported details concerning implementation measurement development or adaptation within educational-, behavioral-, and health studies broadly constructed, including both validated and not validated measurements

iv Reported psychometric and pragmatic properties according to the psychometric and pragmatic evidence rating scale (PAPERS) and measurement equivalence (invariance)

v Measurements which assessed the content aligned with at least one of the Consolidated Framework for Implementation Research (CFIR) domains

Table 2. The Consolidated Framework of Implementation Research (CFIR) with its domains, constructs, and their descriptions* 


\begin{tabular}{|c|c|c|}
\hline Domain & Construct & Description \\
\hline \multirow[t]{8}{*}{$\begin{array}{l}\text { Innovation } \\
\text { Characteristics }\end{array}$} & $\begin{array}{l}\text { Innovation } \\
\text { Source }\end{array}$ & $\begin{array}{l}\text { Whether key stakeholders perceive an intervention as } \\
\text { internally or externally developed. }\end{array}$ \\
\hline & $\begin{array}{l}\text { Evidence } \\
\text { Strength \& } \\
\text { Quality }\end{array}$ & $\begin{array}{l}\text { How the quality and validity of evidence of an intervention are } \\
\text { perceived by stakeholders. }\end{array}$ \\
\hline & $\begin{array}{l}\text { Relative } \\
\text { Advantage }\end{array}$ & $\begin{array}{l}\text { How the advantage of an intervention is perceived by } \\
\text { stakeholders in relation to an alternative solution. }\end{array}$ \\
\hline & Adaptability & $\begin{array}{l}\text { Refers to the degree of which the core components of an } \\
\text { intervention can be adapted and tailored towards local needs. }\end{array}$ \\
\hline & Trialability & $\begin{array}{l}\text { How an intervention can be tested on a small scale, and the } \\
\text { reversibility of its implementation if warranted. }\end{array}$ \\
\hline & Complexity & $\begin{array}{l}\text { How difficult the implementation of an intervention is } \\
\text { perceived to be by stakeholders. This is reflected by duration, } \\
\text { scope, radicalness, disruptiveness, centrality, and intricacy } \\
\text { and number of steps required to implement. }\end{array}$ \\
\hline & $\begin{array}{l}\text { Design Quality \& } \\
\text { Packaging }\end{array}$ & Stakeholders' perception of how an intervention is presented. \\
\hline & Cost & $\begin{array}{l}\text { Costs connected to an intervention such as investments and } \\
\text { supply, as well as the costs of the intervention itself. }\end{array}$ \\
\hline \multirow[t]{4}{*}{ Outer Setting } & $\begin{array}{l}\text { Patient Needs \& } \\
\text { Resources }\end{array}$ & $\begin{array}{l}\text { How well known and prioritized individual needs, including the } \\
\text { barriers and facilitators to meet those needs, are by the } \\
\text { organization. }\end{array}$ \\
\hline & Cosmopolitanism & $\begin{array}{l}\text { How an organization is networked with other (external) } \\
\text { organizations. }\end{array}$ \\
\hline & Peer Pressure & $\begin{array}{l}\text { Refers to the pressure to implement an intervention for } \\
\text { competitive or mimetic reasons among organizations. }\end{array}$ \\
\hline & $\begin{array}{l}\text { External Policy \& } \\
\text { Incentives }\end{array}$ & $\begin{array}{l}\text { Includes a broad content of external strategies to disseminate } \\
\text { interventions, along with policy, regulations, and guidelines } \\
\text { etc. }\end{array}$ \\
\hline \multirow[t]{5}{*}{ Inner Setting } & $\begin{array}{l}\text { Structural } \\
\text { Characteristics }\end{array}$ & $\begin{array}{l}\text { The architecture of an organization, involving size, maturity, } \\
\text { age etc. }\end{array}$ \\
\hline & $\begin{array}{l}\text { Networks \& } \\
\text { Communications }\end{array}$ & $\begin{array}{l}\text { Refers to the nature and quality of formal and informal social } \\
\text { networks and communications in an organization. }\end{array}$ \\
\hline & Culture & An organizations norms and values. \\
\hline & $\begin{array}{l}\text { Implementation } \\
\text { Climate }\end{array}$ & $\begin{array}{l}\text { An organizations capacity and receptivity for change, along } \\
\text { with the reward and support that is given for the use of a } \\
\text { specific intervention. }\end{array}$ \\
\hline & & $\begin{array}{l}\text { This construct contains six additional sub-constructs; Tension } \\
\text { for change, Compatibility, Relative priority, Organizational } \\
\text { incentives \& rewards, Goals and feedback, and Learning } \\
\text { Climate. }\end{array}$ \\
\hline
\end{tabular}

Page 14/17 
Readiness for Implementation

\section{Characteristics} of Individuals
Knowledge \&

Beliefs about the Intervention

Self-efficacy
An organizations' commitment to the decision of the implementation of an intervention.

This construct contains three additional sub-constructs; Leadership engagement, Available resources, and Access to knowledge \& information.

Refers to attitudes and values of individuals in connection to the intervention, as well as their familiarity with content and principles of the intervention.

How individuals perceive their own capabilities to execute the implementation.

Individual Stage

of Change

Refers to the characterization of the stage an individual is in, in relation to their use of the intervention.

Individual

Process Planning
Identification

with Organization

Other Personal Attributes

Engaging

How individuals perceive the organization, as well as their degree of commitment to it.

A broad construct that involves other individual traits.

Refers to the degree of which an intervention and its content for implementation is designed and developed in advance, as well as the quality of the content in that plan.

How individuals are involved in the implementation and use of the intervention.

This construct contains four additional sub-constructs; Opinion leaders, Formally Appointed internal implementation leaders, Champions, and External change agents.

Executing How the implementation actually is carried out, in relation to the plan.

Reflecting \& Evaluation
Feedback about the progress and quality of implementation, and reflections concerning experiences of the implementation.

*Based on the original work of Damschroder and colleagues $(17,44)$.

Table 3. Psychometric and pragmatic domains and their definitions 


\section{Psychometric and pragmatic Domain Definition properties}

PAPERS Scale $(22,35,36)$

Pragmatic criteria
1. Length

2. Language
Number of items

The re-adability of the items included in the measure

3. Cost

The cost researchers pay to use the instrument

4. Assessor burden (Ease of training)

5. Assessor burden (Easy to Interpret)

Psychometric properties criteria

The required training needed for the assessor, and the administration of an instrument

The requirements to interpret the data from a measurement; the complexity of scoring interpretation

Assesses reliability and indicates whether several items that measure the same construct
1. Internal consistency produce similar scores (Cronbach's a)

2. Convergent construct validity

3. Discriminant construct validity

4. Knowngroups validity

5. Predictive criterion validity

6. Concurrent criterion validity related in fact are related (effect size, Cohen's $d$, or correlation, Pearson's $r$ )

The degree to which constructs are theoretically distinct in fact are distinct (effect size, Cohen's $d$, or correlation, Pearson's r)

The extent to which the measure can differentiate groups known to have different characteristics

The degree to which a measurement can predict or correlate with an outcome of interest measured at a future time (Pearson's $r$ )

Assesses whether measurements taken at the same time correlate, and if a measure's
The degree to which constructs are theoretically observed scores correlate with scores from a previously established measure of the construct (Pearson's $r$ )

7. Structural validity

Known as test structure, and refers to the degree a measure's items increase or decrease together (assessed in nine ways*)

8.

Responsiveness

The ability to which a measure can detect clinically important changes over time (Standardized Response Mean=SRM, Pearson's r)

9. Norms
Assesses generalizability based on sample size, means and standard deviations of item values 
Assesses the psychometric equivalence of a construct across groups or measurement occasions, and demonstrates that a construct has the same meaning across groups or across repeated measurements. Measurement invariance is a prerequisite to comparing group means, and is most commonly tested through structural equation modelling (SEM) using confirmatory factor analysis (CFA).

*Normed Fit Index = NFI; Incremental Fit Index = IFl; Goodness of Fit Index = GFl; Tucker-Lewis Index = TLI; Comparative Fit Index = CFI; Relative Non centrality Fit Index = RNI; Standardized RMR = SRMR; Root Mean Square; Error of Approximation $=$ RMSEA; Weighted Root Mean Residual $=W R M R$

\section{Supplementary Files}

This is a list of supplementary files associated with this preprint. Click to download.

- Additionalfile1PRISMAP2015checklistPIMS.pdf 